\title{
Effect of L-lysine in culture medium on nodule formation by bone marrow cells
}

\author{
Masataka Yoshikawa, Yasunori Shimomura, Hideyuki Kakigi, Norimasa Tsuji, Takayoshi Yabuuchi, \\ Hiroyuki Hayashi
}

Department of Endodontics, Osaka Dental University, Osaka, Japan

Email: yosikawa@cc.osaka-dent.ac.jp, shimoyan3@gmail.com, kakigi@cc.osaka-dent.ac.jp, n-tsuji@cc.osaka-dent.ac.jp, takayan0813@yahoo.co.jp, hayashi@cc.osaka-dent.ac.jp

Received 25 July 2012; revised 26 August 2012; accepted 10 September 2012

\begin{abstract}
The aim of this study was to estimate the effect of L-lysine on nodule formation by rat bone marrow cells in vitro. In this study, L-lysine was added to medium for mesenchymal stem cell culture to promote proliferation and differentiation of the cells, and then nodule formation was estimated in an in vitro rat bone marrow cell culture. Bone marrow cells from the bone shafts of the femora of Fischer 344 rats were cultured in minimum essential medium with $20 \mu \mathrm{l}$ of L-lysine solution at $10^{-4}, 10^{-5}, 10^{-6}, 10^{-7}$ or $10^{-8} \mathrm{M}$. Dexamethasone was also added to the medium at 10 nM for differentiation of stem cells from bone marrow into osteoblast progenitor cells. The subculture was performed for 2 weeks. The quantity of osteocalcin in rat bone marrow cell culture with dexamethasone was $392 \mathrm{ng} / \mathrm{ml}$. In the medium with dexamethasone and $10^{-8} \mathrm{M}$ L-lysine, the quantity of osteocalcin was $437 \mathrm{ng} / \mathrm{ml}$. Nodules only formed upon addition of $20 \mu \mathrm{l}$ of L-lysine at $10^{-8} \mathrm{M}$. It was indicated that $10^{-8} \mathrm{M}$ L-lysine should be the optimal concentration for calcification. For nodule formation by rat bone marrow cells in vitro, the optimum concentration of L-lysine in culture medium might be $20 \mu \mathrm{l}$ of $10^{-8} \mathrm{M}$. L-lysine could play an important role in matrix production for bone formation in vitro.
\end{abstract}

Keywords: L-Lysine; Bone marrow Cells; Calcium Deposition; Dexamethasone; In Vitro

\section{INTRODUCTION}

A definitive goal of regenerative therapy in dentistry is restoration of a partial defect of a tooth. In addition, for a missing tooth, regeneration of the whole tooth by tissue engineering is another goal. Studies to regenerate a whole tooth have proceeded by different methods [1-3]. However, many studies over a long period appear neces- sary before the actual utilization of regenerated teeth produced by tissue engineering. For tooth regeneration, stem cells as progenitor cells or odontoblasts are rquired. It is considered that the most desirable source of cells may be the dental pulp. However, there are not many opportunities to extract teeth. We can only obtain dental pulp from sound teeth which must be extracted owing to orthodontic requirements. Moreover, the isolation of odontoblasts from dental pulp may be too difficult. Methods to differentiate cells in the dental pulp into odontoblasts or stem cells have not been established. Attempts have been made to regenerate teeth partially or totally [4-8]. The development of a removed tooth germ to a mature tooth in vitro was also attempted [9]. However, realization of the regeneration or restoration of a tooth by techniques of tissue engineering may be difficult in the near future.

For the regeneration of a tooth, it is desirable to create a dentine-pulp complex using odontoblasts differentiated from stem cells. The use of scaffolds may also be desirable for the reconstitution of a whole tooth or part of a tooth because of the three-dimensional structure. We attempted to produce bone-like tissue using bone marrow cells in porous hydroxyapatite (HA) scaffolds [10]. However, a large number of undifferentiated mesenchymal stem cells are necessary for bone formation in all pores in an HA scaffold. Mesenchymal stem cells from bone marrow must be used in an in vitro or in vivo study until stem cells are isolated from a tissue in the oral cavity as a source of such cells for tooth regeneration.

Mesenchymal stem cells can differentiate into many types of functional mesenchymal cells under specific extracellular conditions [11]. It was also reported previously that stem cells could be easily differentiated into osteoblasts, chondrocytes, adipocytes or myocytes using various growth and differentiation-inducing factors [12, 13]. Therefore, it is desirable to activate and promote the proliferation of a small number of stem cells by the addition of supplements to the culture medium. 
The formation of bone-like tissue by osteoblasts was facilitated by the effects of some amino acids [14]. In this study, we selected L-lysine (Lys) to assess the effect. Lys has been shown to be effective in the healing process to improve bone fractures [15]. Lys also affects the bone status in metabolic diseases such as primary and secondary osteoporosis, in which an imbalance between the anabolic activity of osteoblasts and bone resorption by osteoclasts is the cause of bone rarefaction [16]. In this study, the effectiveness of Lys for bone formation as a promoter of mesenchymal stem cell proliferation and differentiation was assessed in an in vitro bone marrow cell culture.

\section{MATERIALS AND METHODS}

\subsection{Rat Bone Marrow Cell Isolation and Preparation of the Cell Suspension}

This study was performed under the Guidelines for Animal Experimentation at Osaka Dental University. Regarding the use and care of the animals, the Animal Welfare Committee of Osaka Dental University approved the experimental procedures.

Bone marrow cells were obtained from the bone shafts of the femora of 6-week-old male Fischer 344 (Clea Japan, Osaka, Japan) rats after euthanasia by halothane suffocation. The cells were flushed out using $10 \mathrm{ml}$ of culture medium expelled from a syringe with a 21-gauge needle. Primary culture of the cells was performed for 1 week in a cell culture flask (T-75; BD Biosciences, MA, USA) containing minimum essential medium (MEM; Nacalai Tesque, Inc., Kyoto, Japan) supplemented with $15 \%$ fetal bovine serum (FBS; JRH Biosciences, KS, USA) and antibiotics (100 U/ml penicillin, $100 \mathrm{mg} / \mathrm{ml}$ streptomycin and $0.25 \mathrm{mg} / \mathrm{ml}$ amphotericin B, SigmaAldrich Co., MO, USA). The medium was changed 2 times.

After primary culture, bone marrow cells in T-75 culture flask were washed three times using phosphate buffer solution without $\mathrm{Ca}^{2+}$ and $\mathrm{Mg}^{2+}$ (PBS (-); Nacalai Tesque Inc.) and the cells were isolated from the bottom of a T-75 flask with trypsin-EDTA solution $(0.5 \mathrm{mg} / \mathrm{ml}$ trypsin and $0.53 \mu \mathrm{mol}$ EDTA; Nacalai Tesque Inc.) to prepare bone marrow cell suspension. Harvested cells were re-suspended in culture medium at $1 \times 10^{5}$ cells $/ \mathrm{ml}$.

\subsection{Nodule Formation by Rat Bone Marrow Cell Culture in Vitro}

The bone marrow cell suspension at $1 \times 10^{5}$ cells $/ \mathrm{ml}$ was prepared in MEM. A cell suspension with a volume of 2 $\mathrm{ml}$ was poured into each well of 6-well culture plates (BD Biosciences) for bone marrow cell seeding. For 2 weeks, subculture was performed with MEM containing
15\% FBS, 1 mM $ß$-glycerophosphate ( $ß$-GP; EMD Biosciences Inc., CA, USA), and antibiotics. The medium was renewed three times a week.

Lys (Wako Pure Chemical Industries, Ltd., Osaka, Japan) solutions at $10^{-8}, 10^{-7}, 10^{-6}, 10^{-5}$ and $10^{-4} \mathrm{M}$ were prepared. Culture medium with a volume of $2 \mathrm{ml}$ containing $1 \times 10^{5}$ bone marrow cells in the wells was supplemented with $20 \mu$ of each concentration of Lys solution. To promote bone marrow cell differentiation and calcified nodule formation, $10 \mathrm{nM}$ dexamethasone (Dex; ICN Pharmaceutical Inc., CA, USA) and $82 \mathrm{mg} / \mathrm{ml}$ ascorbic acid (Vc; Sigma-Aldrich Co.) were also added. Nodule formation was confirmed under a phase contrast inverted microscope.

\subsection{Measurement of Alkaline Phosphatase Activity Level and Osteocalcin Production in Rat Bone Marrow Cell Culture}

Each culture medium in all wells was collected for quantitative analysis of osteocalcin produced during subculture of bone marrow cells. Then, each subcultured cell layer on the plate was scraped off in $1 \mathrm{ml}$ of $0.05 \mathrm{M}$ sodium phosphate buffer including $2 \mathrm{mM}$ EDTA and $2 \mathrm{M}$ $\mathrm{NaCl}$. The cells in the buffer solution were homogenized and $0.1 \mathrm{ml}$ of sonicated cell suspension was used for DNA measurement. Salmon sperm DNA (Life Technologies Inc., CA, USA) was used as the standard. DNA measurements were performed using the fluorescence emission at $460 \mathrm{~nm}$ in the presence of $0.5 \mu \mathrm{g} / \mathrm{ml}$ Hoechst 33,258 (Wako Pure Chemical Industries Ltd.).

The other sonicated cell suspension was used for measurement of ALP activity level. The suspension was centrifuged at $16,000 \times \mathrm{g}$ for 1 minute. For measurement of ALP activity, $0.1 \mathrm{ml}$ of $p$-nitrophenyl phosphate (PNP; Zymed Lab, CA, USA) was added as substrate in $20 \mu \mathrm{l}$ of the supernatant and incubated at $37^{\circ} \mathrm{C}$ for 30 minutes. The reaction was stopped by addition of $100 \mu \mathrm{l}$ of $\mathrm{NaOH}$ at $0.2 \mathrm{M}$ concentration. The quantity of $p$-nitrophenol produced by reaction of the supernatant with PNP was determined by measurement of the absorbance at a wavelength of $405 \mathrm{~nm}$. The ALP level of the cultured bone marrow stem cells with and without addition of Lys in the culture medium was represented as $\mu \mathrm{M}$ p-nitrophenol released after 30 minutes of incubation at $37^{\circ} \mathrm{C}$. The ALP/DNA ratio was calculated as ALP activeity.

Supernatant in wells of all culture plates was collected at 14 days. The quantity of osteocalcin (OC) in the supernatant was measured immunochemically (Rat Osteocalcin ELISA kit DS, DS Pharma Biomedical Co., Ltd., Osaka, Japan). Each supernatant at a volume of $15 \mu \mathrm{l}$ from the culture media was poured into each well of a 96-well microplate to react with anti-rat OC antibody 
which was coated on the bottom of the wells. Peroxidase-conjugated anti-rat osteocalcin polyclonal antibody was added in each well. Equal amounts of peroxidase substrate and hydrogen peroxide water was also added in each well followed by incubation in the dark. Absorbance at $450 \mathrm{~nm}$ was measured using a spectrophotometer (SPECTRA max PLUS; Nihon Molecular Devices Corporation, Tokyo, Japan).

Significant differences in the ALP activity in the bone marrow cell differentiation and the quantity of osteocalcin produced during nodule formation by bone marrow cells in culture medium were statistically analyzed between cases with and without the addition of each concentration of Lys. These results are presented as mean and standard deviation. Statistical comparisons between the mean values of ALP activity and quantity of osteocalcin were performed using two-way unrepeated ANOVA followed by post hoc analysis using TukeyKramer's test. Differences of $p<0.01$ were considered significant.

\subsection{Von Kossa's Staining}

Calcified products of the cells were stained by von Kossa's silver nitrate method. The methods of the staining were briefly as follows. After fixation of the cells on the bottom of wells, calcified product in the wells was soaked in 5\% silver nitrate solution for 60 minutes. Then, under sunlight, the product was reacted with $5 \%$ sodium thiosulfate (Wako Pure Chemical Industries, Ltd.). Moreover, the cells were counterstained with Kernechtrot solution for 5 minutes.

\section{RESULTS}

\subsection{Nodule Formation by Rat Bone Marrow Cells in Vitro}

In each well of 6-well culture plates supplemented with or without Lys solutions, rat bone marrow cells were cultured for 2 weeks. Nodule was formed in the wells supplemented with Lys solution at each concentration (Figure 1(a)). However, nodule was not observed on rat bone marrow cell culture without Dex in MEM (Figure 1(b)).

\subsection{Alkaline Phosphatase Activity and Osteocalcin Production in Medium by Rat Bone Marrow Cell Culture}

The result of the measured ALP/DNA ratio is shown in Figure 2. The level of ALP activity of the cells cultured in the medium with Dex was $0.131 \mu \mathrm{M} / \mu \mathrm{g}$ DNA, and the cells cultured with Dex and $10^{-8} \mathrm{M}$ Lys showed 0.204 $\mu \mathrm{M} / \mu \mathrm{g}$ DNA. Addition of Dex to culture medium induced a high level of ALP activity in each of the bone

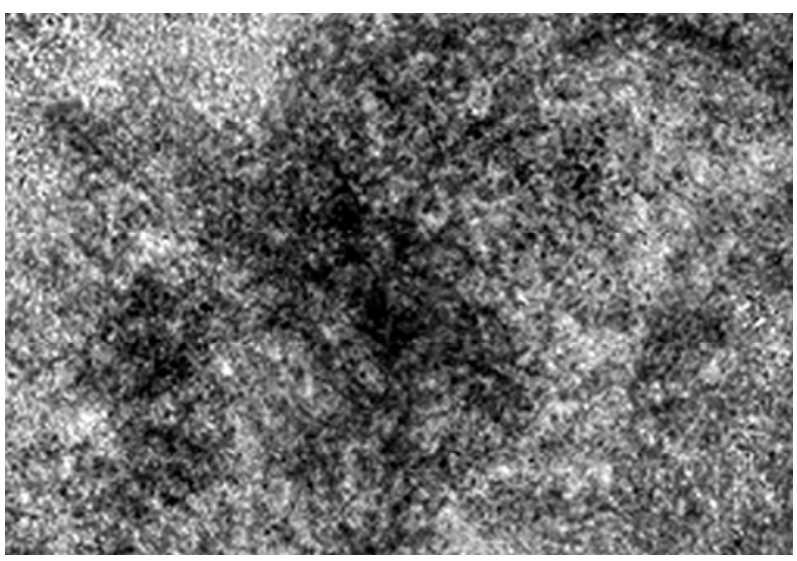

(a)

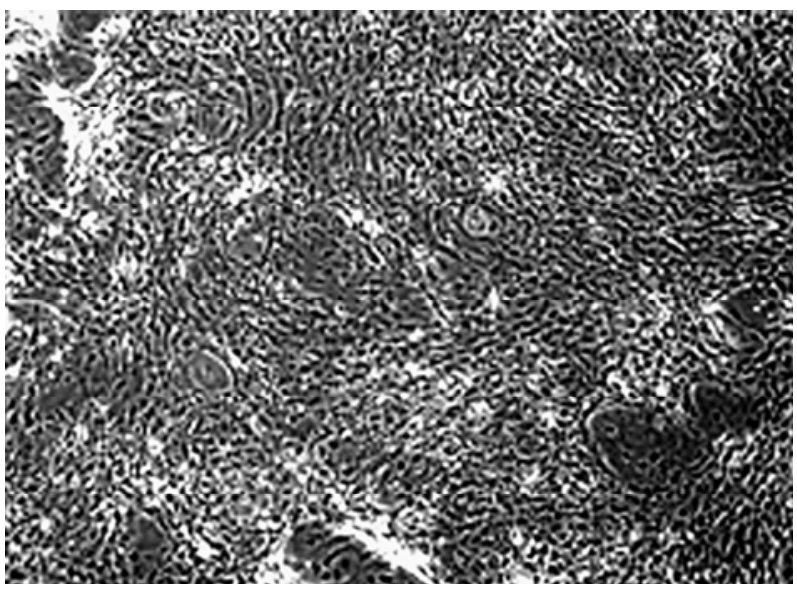

(b)

Figure 1. Phase contrast inverted microscopic image of rat bone marrow cell culture in MEM. (a) Nodule formed by rat bone marrow cells cultured in MEM with dex and a volume of $20 \mu \mathrm{l}$ Lys solutions at $10^{-5} \mathrm{M}$; (b) Nodule is not observed in rat bone marrow cell culture without Dex.

marrow cell cultures with and without Lys. It was shown by measuring the level of ALP/DNA ratio of bone marrow cells that Lys itself had no effect on the activation of bone marrow cell differentiation in the culture. However, the effect of Dex to activate the cell differentiation was enhanced by the addition of Lys to the culture medium. The level of ALP activity significantly increased in the bone marrow cell culture medium with Lys compared with that without Lys $(p<0.01)$. There was no significant difference in the level of ALP activity between each concentration of Lys added to the cell culture medium.

The quantity of osteocalcin measured in culture medium is also shown in Figure 3. The quantity of osteocalcin in bone marrow cell culture with Dex was 392 $\mathrm{ng} / \mathrm{ml}$. The quantity of osteocalcin in the medium with Dex and $10^{-8}$ M Lys was $437 \mathrm{ng} / \mathrm{ml}$. The level of osteocalcin product was significantly higher in the medium with Lys at $10^{-8}, 10^{-7}, 10^{-6}, 10^{-5}$ or $10^{-4} \mathrm{M}$ than in that without Lys $(p<0.005)$. 


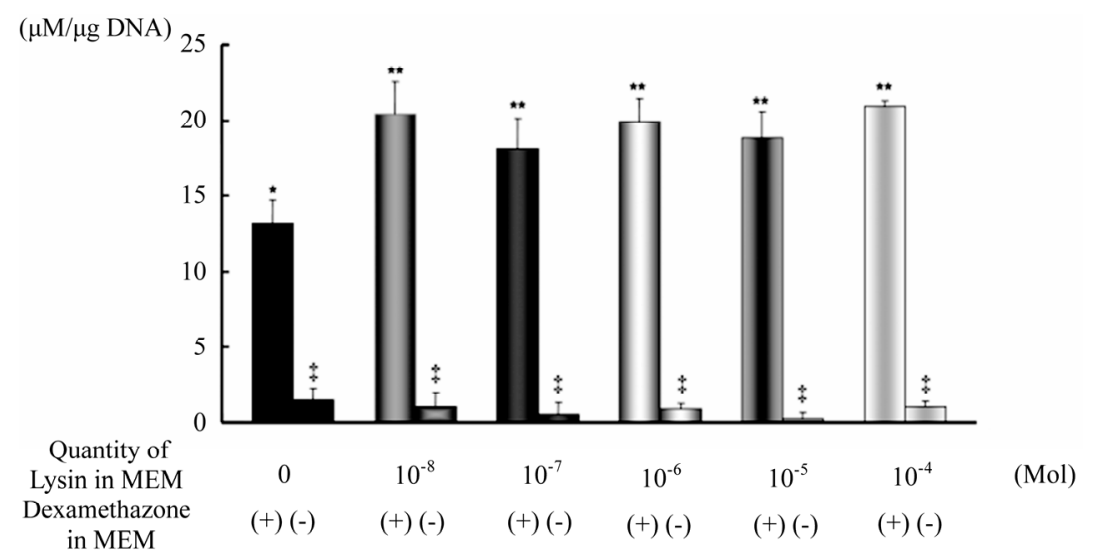

Figure 2. Alkaline phosphatase activity level of rBMCs cultured in MEM with and without Lys Values are means \pm SD $(n=5)$. Upon comparison of alkaline phosphatase activity level between rBMCs cultured in MEM with or without Lys, there was greater activity in the rBMCs cultured in MEM with Lys than in that without Lys. ${ }^{*} p<0.01$ vs. ${ }^{*}$. The level of alkaline phosphatase activity was significantly higher in rBMCs cultured in MEM with Dex. $*$ and $* * p<0.005$ vs. $\$$.

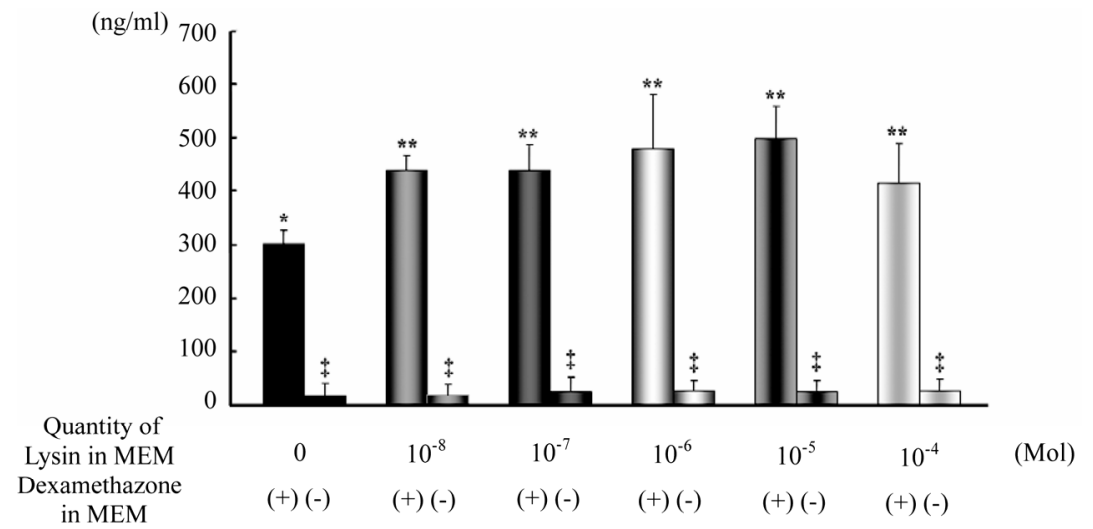

Figure 3. Quantity of osteocalcin measured in rBMCs cultured in MEM with and without Lys quantities of osteocalcin measured in rBMCs cultured in MEM with Lys were significantly greater than those in rBMCs cultured without Lys. ${ }^{* *} p<$ 0.01 vs. *. The level of osteocalcin was higher in rBMCs cultured in MEM with Dex. ${ }^{*}$ and $* * p<0.01$ vs. $\ddagger$.

\subsection{Von Kossa's Staining}

After 2 weeks of bone marrow cell subculture in MEM with $\mathrm{Vc}, \beta$-GP and Dex, remarkable staining was recognized as shown in the illustration of von Kossa's staining (Figure 4). On the well bottoms of culture plates both with and without Lys, the staining showed conspicuous calcium deposition by bone marrow cells being cultured in MEM containing Vc, $\beta$-GP and Dex. Bone marrow cell culture on all wells in MEM without Dex induced less calcium deposition whether Lys was added or not.

\section{DISCUSSION}

It is considered that regeneration of the hard tissue component in tooth construction will be achieved by osteogenesis using stem cells in the pores of a porous scaffold.
Multifunctional undifferentiated mesenchymal stem cells from bone marrow and periosteum have the capability to differentiate into chondroblasts, adipocytes or osteoblasts [17]. Moreover, it was recently suggested that these stem cells show phenotypes of endothelial cells, neurocytes, smooth muscle cells and myocardial cells [12]. Attempts have been made to use mesenchymal bone marrow stem cells for osseous regeneration $[7,8,18]$ and osseous healing [9]. The use of undifferentiated mesenchymal stem cells in pulp tissue may be desirable for tooth regeneration. In fact, it was reported that stem cells isolated from tooth pulp differentiate into hepatocytes, fibroblasts and osteoblasts [19].

However, only a small number of stem cells would be provided from a tooth because of the small volume of the dental pulp in each human tooth. Moreover, the number 

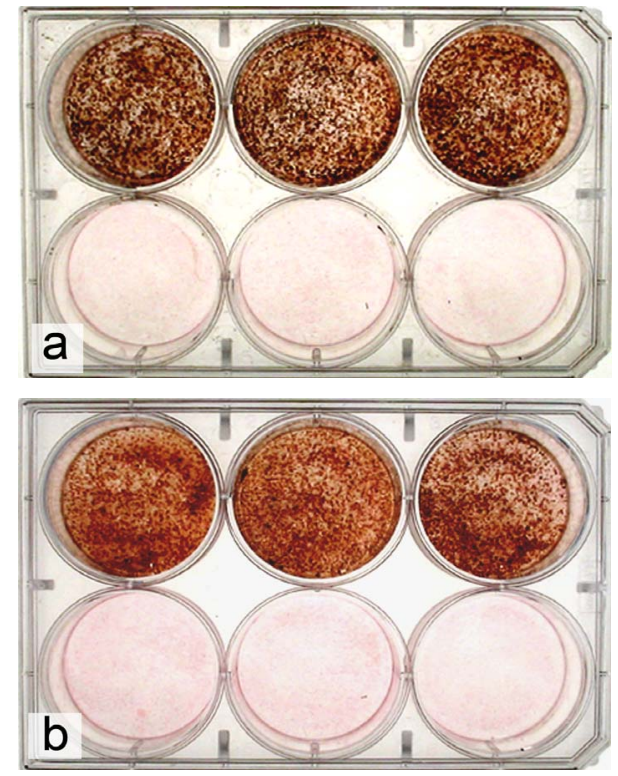

Figure 4. Images of von Kossa's staining of nodule formed by bone marrow cell culture after 2 weeks. Upper wells: Nodule is formed by rat bone marrow cells cultured in MEM with dex. Under wells: Nodule is not formed by rat bone marrow cell culture without dex. (a) Left wells: Without Lys. Center wells: With a volume of 2 $\mathrm{ml}$ Lys at $10^{-8} \mathrm{M}$. Right wells: With a volume of $2 \mathrm{ml}$ Lys at $10^{-7} \mathrm{M}$; (b) Left wells: With a volume of $2 \mathrm{ml}$ Lys at $10^{-6} \mathrm{M}$. Center wells: With a volume of $2 \mathrm{ml}$ Lys at $10^{-5} \mathrm{M}$. Right wells: With a volume of $2 \mathrm{ml}$ Lys at $10^{-4} \mathrm{M}$.

of human permanent teeth ranges from 28 to 32 . There is a limit to the amount of dental pulp to be available for regeneration of a whole tooth or part of a tooth. It is impossible to extirpate dental pulp from most of the sound teeth. It is known that a layer of odontoblasts is present along the wall of pulp cavity and that these cells contribute to dentine formation. It is generally difficult to isolate the cells from the pulp cavity. Moreover, there are not so many undifferentiated mesenchymal stem cells in the pulp tissue. It may also be disadvantageous to remove pulp tissue from an intact tooth. In dentistry, a source of undifferentiated mesenchymal stem cells is required for tooth regeneration. The cells had to be harvested from bone marrow of experimental animals in this in vitro examination. The efforts to attach many stem cells and to differentiate stem cells effectively into osteoblasts in the pores of porous scaffolds should be made. It is considered that one of the important factors for bone formation in the pores of scaffold by stem cells is adhesion of a large number of the cells on the walls of pores. Several in vitro and in vivo studies suggested that osteoblasts activated by Lys might positively affect to bone metabolism $[8,10,20]$.

In this in vitro study, it was shown that Lys can modulate the growth and the differentiation of osteoblasts cultured with Dex. There is the receptor on the cell surface or in the cytoplasm to Lys [21]. Furthermore, all amino acids have been shown to present mitogenic activity at doses equivalent to $0.1-, 1$ - and 10-fold their plasma concentration [8]. It is possible for them to regulate cell functions by signal transduction mediated receptors. In this study, nodule formation by bone marrow cells was significantly facilitated and osteocalcin production increased in the medium with a concentration of $10^{-8} \mathrm{M}$ of Lys. It was thought that $10^{-8} \mathrm{M}$ Lys had a direct effect on the undifferentiated mesenchymal stem cells among the bone marrow cells.

Some cytokines seem to play an important role in the pathogenesis of senile and post-menopausal bone loss because of their action as local mediators of bone remodeling [22]. Insulin-like growth factor-1 (IGF-1) is an important regulator of osteoblast metabolism and differentiation, and enhances the synthesis of bone matrix proteins by cultured osteoblastic cells [23]. It has been reported that the effects of Lys are probably mediated by IGF-1, which stimulates osteoblast proliferation and differentiation, type 1 collagen synthesis, OC production, and ALP activity [6,9]. Moreover, IGF-1 is considered as an important factor for bone longitudinal growth [11] and plays a role in trabecular and cortical bone formation. Lys increase in vitro IGF-1 production by osteoblast cultures [8]. Alternative approaches directed at increaseing IGF-1 production of osteoblasts seem to be attractive. Therefore, it would be interesting to verify the effects of Lys on IGF-1 expression and production by osteoblasts.

\section{CONCLUSION}

It was shown that $10^{-8} \mathrm{M}$ Lys should be a beneficial concentration for calcification by bone marrow cells in vitro. Lys might induce differentiation of multi-potent bone marrow stem cells to osteoblast precursor cells followed by nodule formation in the culture. It is concluded that Lys could play an important role in matrix production for bone formation in vitro.

\section{ACKNOWLEDGEMENTS}

This study was performed at the Morphological Research Facilities, Tissue Culture Facilities, Laboratory and Animal Facilities, Institute of Dental Research, Osaka Dental University.

This study was supported in part by a 2008-2010 Grant-in-Aid for Scientific Research (C: 20592246) and a 2011-2013 Grant-in-Aid for Scientific Research (C: 23592820) from the Japan Society for the Promotion of Science.

\section{REFERENCES}

[1] Ida-Yonemochi, H., Nakatomi, M., Harada, H., Takata, 
H., Baba, O. and Ohshima, H. (2011) Glucose uptake mediated by glucose transporter 1 is essential for early tooth morphogenesis and size determination of murine molars. Developmental Biology, 363, 52-61.

[2] Traphagen, S. and Yelick, P.C. (2009) Reclaiming a natural beauty: Whole-organ engineering with natural extracellular materials. Regenerative Medicine, 4, 747-758.

[3] Thesleff, I. and Tummers, M. (2003) Stem cells and tissue engineering: Prospects for regenerating tissues in dental practice. Medical Principles and Practice, 12, 4350 .

[4] Caplan, A.I. and Bruder, S.P. (2001) Mesenchymal stem cells: Building blocks for molecular medicine in the $21 \mathrm{st}$ century. Trends in Molecular Medicine, 7, 259-264. doi:10.1016/S1471-4914(01)02016-0

[5] Derubeis, A.R. and Cancedda, R. (2004) Bone marrow stromal cells (BMSCs) in bone engineering: Limitations and recent advances. Annals of Biomedical Engineering, 32, 160-165.

[6] Canalis, E., McCarthy, T.L. and Centrella, M. (1991) Growth factors and cytokines in bone cell metabolism. Annual Review of Medicine, 42, 17-24.

[7] D'Ippolito, G., Schiller, P.C., Ricordi, C., Roos, B.A. and Howard, G.A. (1999) Age-related osteogenic potential of mesenchymal stromal stem cells from human vertebral bone marrow. Journal of Bone and Mineral Research, 14, 1115-1122.

[8] Chevalley, T., Rizzoli, R., Manen, D., Caverzasio, J. and Bonjour, J.P. (1998) Arginine increases insulin-like growth factor-I production and collagen synthesis in osteoblastlike cells. Bone, 23, 103-109.

[9] Baylink, D.J., Finkelma, R.D. and Mohan, S. (1993) Growth factors to stimulate bone formation. Journal of Bone and Mineral Reseach, 8, 565-572. doi:10.1002/jbmr.5650081326

[10] Yoshikawa, M., Tsuji, N., Shimomura, Y., Hayashi, H. and Ohgushi, H. (2008) Osteogenesis depending on geometry of porous hydroxyapatite scaffolds. Clcified Tissue International, 83, 139-145. doi:10.1007/s00223-008-9157-y

[11] Pittenger, M.F. and Martin, B.J. (2004) Mesenchymal stem cells and their potential as cardiac therapeutics. Circulation Research, 95, 9-20. doi:10.1161/01.RES.0000135902.99383.6f

[12] Froesch, E.R., Schmid, C., Schwander, J. and Zapf, J. (1985) Actions of insulin-like growth factors. Annual Review of Physiology, 47, 443-467. doi:10.1146/annurev.ph.47.030185.002303

[13] Fiore, C.E., Pennisi, P., Cutuli, V.M., Prato, A., Messina, R. and Clementi, G. (2000) L-arginine prevents bone loss and bone collagen breakdown in cyclosporin A-treated rats. European Journal of Pharmacology, 408, 323-326. doi:10.1016/S0014-2999(00)00800-1

[14] Torricelli, P., Fini, M., Giavaresi, G. and Giardino, R (2003) Osteoblasts cultured from osteoporotic bone: A comparative investigation on human and animal-derived cells. Artificial Cells, Blood Substitutes and Biotechnology, 31, 263-277. doi:10.1081/BIO-120023157

[15] Torricelli, P., Fini, M., Giavaresi, G. and Giardino, R. (2001) Bone tissue cultures: An in vitro model for the evaluation of bone defect healing after L-arginine and L-lysine administration. Artificial Cells, Blood Substitutes and Biotechnology, 29, 325-334. doi:10.1081/BIO-100104234

[16] Visser, J.J. and Hoekman, K. (1994) Arginine supplementation in the prevention and treatment of osteoporosis. Medical Hypotheses, 43, 339-342. doi:10.1016/0306-9877(94)90113-9

[17] Barry, F.P. and Murphy, J.M. (2004) Mesenchymal stem cells: Clinical applications and biological characterization. The International Journal of Biochemistry \& Cell Biology, 36, 568-584. doi:10.1016/j.biocel.2003.11.001

[18] Petite, H., Viateau, V., Bensaïd, W., Meunier, A., De Pollak, C., Bourguignon, M., Oudina, K., Sedel, L. and Guillemin, G. (2000) Tissue-engineered bone regeneration. Nature Biotechnology, 18, 959-963. doi:10.1038/79449

[19] Ikeda, E., Yagi, K., Kojima, M., Yagyuu, T., Ohshima, A., Sobajima, S., Tadokoro, M., Katsube, Y., Isoda, K., Kondoh, M., Kawase, M., Go, M.J., Adachi, H., Yokota, Y., Kirita, T. and Ohgushi, H. (2008) Multipotent cells from the human third molar: Feasibility of cell-based therapy for liver disease. Differentiation, 76, 495-505. doi:10.1111/j.1432-0436.2007.00245.x

[20] Conconi, M.T., Tommasini, M., Muratori, E. and Parnigotto, P.P. (2001) Essential amino acids increase the growth and alkaline phosphatase activity in osteoblasts cultured in vitro. II Farmaco, 56, 755-761. doi:10.1016/S0014-827X(01)01126-0

[21] Mostaqul-Huq, M.D., Tsai, N.P., Khan, S.A. and Wei, L.N. (2007) Lysine trimethylation of retinoic acid recaptor-alpha: A novel means to regulate receptor function. Molecular \& Cellular Proteomics, 6, 677-688. doi:10.1074/mcp.M600223-MCP200

[22] Marie, P. (1997) Growth factors and bone formation in osteoporosis: Roles for IGF-I and TGF-beta. Revue du Rhumatisme, 64, 44-53.

[23] Tumber, A., Meikle, M.C. and Hill, P.A. (2000) Autocrine signals promote osteoblast survival in culture. Journal of Endocrinology, 167, 383-390. doi:10.1677/joe. 0.1670383 